Mosier-Boss, P.A. and S. Szpak, The Pd/(n)H system: transport processes and development of thermal instabilities. Nuovo Cimento Soc. Ital. Fis. A, 1999. 112: p. 577.

\title{
The $\mathrm{Pd} /{ }^{n} \mathrm{H}$ system: Transport processes and development of thermal instabilities
}

\author{
P. A. Mosier-Boss and S. Szpak \\ Spawar Systems Center San Diego-San Diego, CA 92152-5001, USA
}

(ricevuto il 2 Dicembre 1998; revisionato il 24 Maggio 1999; approvato il 15 Giugno 1999)

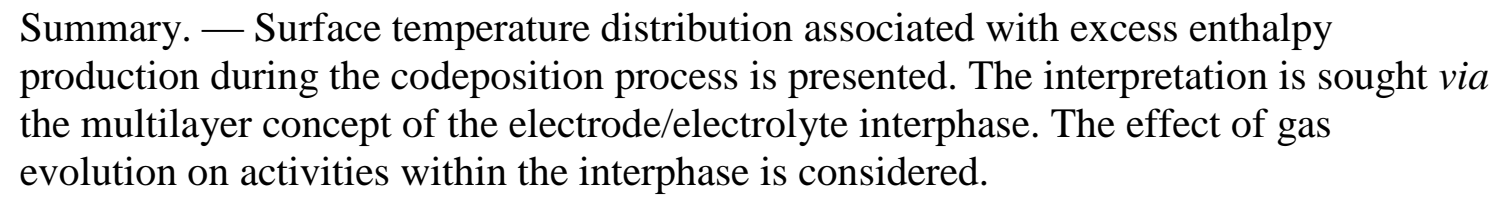

PACS 28.90 - Other topics in nuclear engineering and nuclear power studies.

Recent years have witnessed renewed interest in the hydrogen economy, with emphasis on the metal/hydrogen system and its application to energy conversion efforts. Two industrially important aspects are emphasized, viz. the amount of hydrogen stored and the rate at which it can be transferred (absorbed/desorbed) because they are crucial factors in the design and construction of electrochemical energy conversion devices. The March 1989 announcement of excess enthalpy generation [1], the Fleischmann-Pons effect, provided an added incentive to examine in greater detail the behavior of the $\mathrm{Pd} / \mathrm{D}$ system at very high electrode loadings and at high cell currents.

In an earlier modeling of transport processes, we adopted a multilayer concept of the interphase, formulated transport equations based on the conservation of charge and matter and analyzed events occurring in proximity to the electrode/solution contact surface as a function of the cell current for a set of rate constants characterizing the hydrogen evolution reaction [2]. This model is employed here in the analysis of thermal events associated with the $\mathrm{Pd}+\mathrm{D}$ codeposition.

\section{1. - Thermal events during $P d+D$ codeposition}

Thermal events occurring during the electrochemical compression of the $\mathrm{Pd} / \mathrm{D}$ system are usually discussed in terms of excess enthalpy produced over a period of time, often several days. Thus, they represent an average value and provide no information on the distribution of heat sources. However, when the surface of the electrode producing excess enthalpy is viewed with an infra-red camera, the presence of randomly distributed (in time and space) heat sources of short duration is revealed which subsequently merge to form larger oscillating islands. An example of hot spots recorded in the early stages of 
codeposition is illustrated in figs, la and b. In this example, a Ni screen, placed close to the very thin cell wall (Mylar film), served as the negative electrode, upon which a deuterium-saturated Pd film was formed by the codeposition process $[3,4]$. Such electrodes exhibit a "carpet-like" appearance, i.e., the structure that assures locally a nonuniform distribution of current density and/or overpotentials as well as very rapid absorption of deuterium. Simultaneous evolution of gas bubbles introduces an added component, viz. a source of random distribution of localized gradients (electrical, mechanical and chemical).
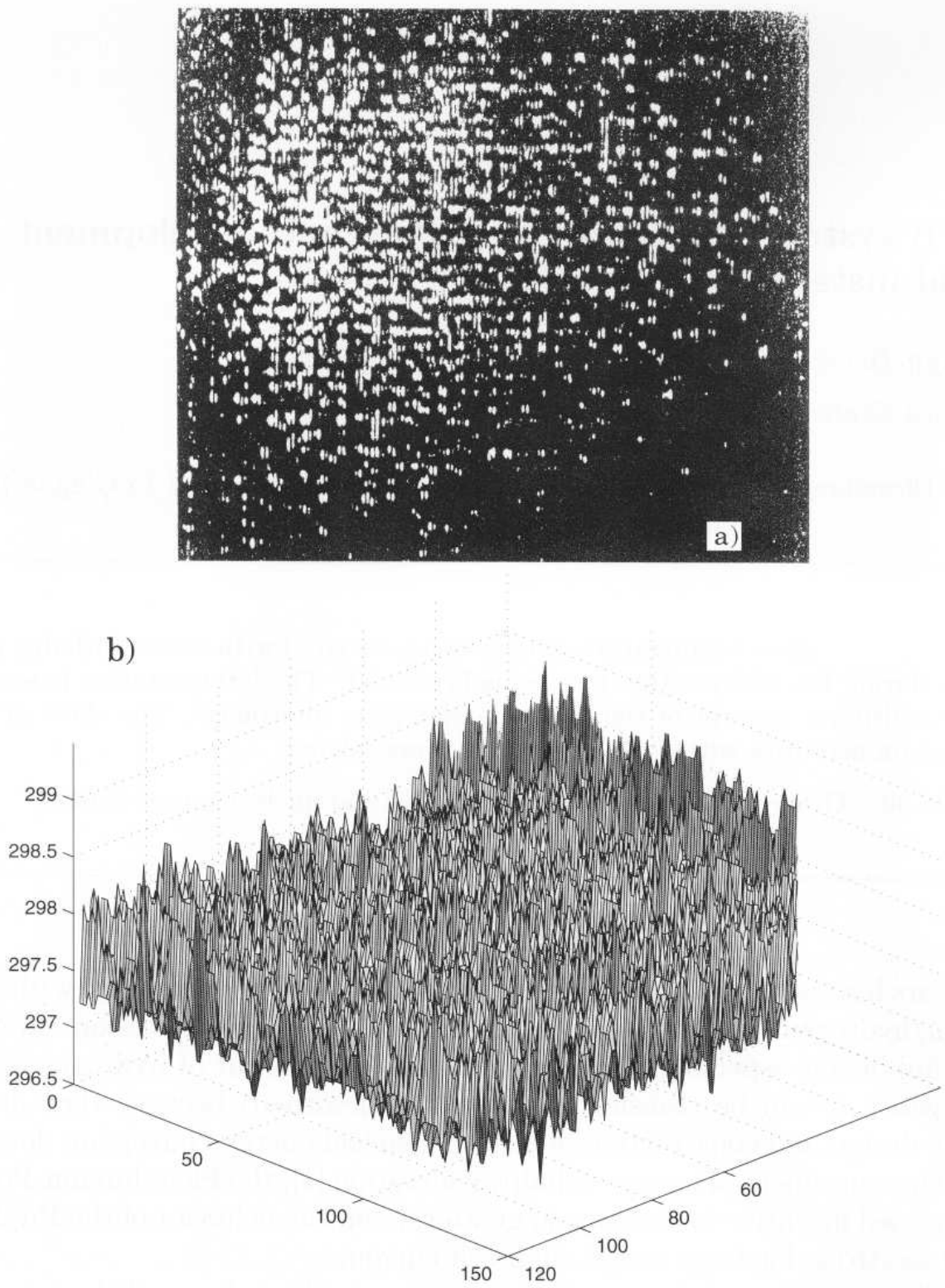

Fig. 1. - Infra-red photographs of the electrode surface, a) View perpendicular to the electrode surface showing distribution of hot spots, b) View parallel to the electrode surface showing temperature gradients. Photographs taken by infra-red camera manufactured by Lynx Real-Time Systems, Los Gatos, CA 95030. 
Qualitatively, judging from the steep temperature gradients, fig. 1b, heat sources are of high intensity and located very close to the contact surface. Evidently, the subsurface region of the active $\mathrm{Pd} / \mathrm{D}$ system consists of domains of high deuterium concentration (activity). Once formed; these domains are unstable. During current flow they are the source of excess enthalpy generation - in the absence of current flow they generate an outflow of deuterium.

\section{2. - Development of thermal instabilities}

It is known that processes which are stable in contacting bulk phases may become unstable in the interphase region and vice versa. The development of instabilities and/or oscillatory behavior is expected in highly nonlinear systems, of which an operating electrochemical cell is a prime example $[5,6]$. The development of such instabilities was discussed by Fleischmann et al. [7,8]. They postulated that production of enthalpy in an operating $\mathrm{Pd} / \mathrm{D}_{2} \mathrm{O}$ cell can be expressed as a function of a set of time/space-dependent variables and their differentials,

$$
Q=\Phi(E, \mathrm{~d} E, \eta, \mathrm{d} \eta, \theta, X, \mathrm{~d} X, \gamma, \mathrm{d} \gamma)
$$

These variables are: cell voltage, $E$, cathodic overpotential, $\eta$ (in particular its Volta component) on the solution side of the interphase, surface coverage $\theta$, deuterium content, $X$ (expressed as the $\mathrm{D} / \mathrm{Pd}$ atomic ratio), and the formation of the $\gamma$-phase. They noted that this functional dependence may lead to a number of features, among them the development of local instabilities. Indeed, the development of such instabilities, resulting in localized hot spots, was reported by us previously [9].

\section{3. - Discussion}

Some of the variables in eq. (1) can be controlled by the experimenter, others cannot. Because these variables are interconnected, they can lead to the development of instabilities. To get a clearer picture of the events leading to the development of instabilities, we examine the following: i) the structure of the interphase, ii) the nature of adsorbed species, iii) the development of an active interphase and iv) interphase relaxation.

3.1. Structure of electrodelelectrolyte interphase. - The electrode/electrolyte interphase is an assembly of non-autonomous layers whose structure is determined by operating processes [10]. The interface is defined as the contact surface (surface of discontinuity). This view is adopted because otherwise the interphase would have to be considered a single element, i.e., the heterogeneous nature of the real interphase would have to be disregarded.

Transport of electrochemically generated hydrogen across the interphase is due to the coupling of interfacial processes: $(b) \rightarrow\left(r_{1}\right) \rightarrow(a d) \rightarrow\left(\lambda_{2}\right)$ and the transport of interstitials: $\left(\lambda_{2}\right) \rightarrow(m)$ in the electrode interior [3]. The processes of interest during charging are: reduction of $\mathrm{H}^{+}, \mathrm{D}^{+}$ions $/ \mathrm{H}_{2} \mathrm{O}, \mathrm{D}_{2} \mathrm{O}$ molecules occurring within the charge transfer layer, $\left(r_{1}\right)$, reactions (i) and (ii):

(i) $\mathrm{H}_{(r 1)}^{+}+\mathrm{e}_{(r 1)}^{-} \leftrightarrow \mathrm{H}_{(\mathrm{ad})} ; j_{1}$, 


$$
\mathrm{H}^{+}(r 1)+\mathrm{H}_{(\mathrm{ad})}+\mathrm{e}_{(r 1)}^{-} \leftrightarrow \mathrm{H}_{2,(g)} ; j_{2} .
$$

The adsorbed hydrogen restructures the electrode surface and either leaves the electrode surface by forming gas bubbles, reaction (iii), or enters the subsurface region. $\left(\lambda_{2}\right)$, reaction iv):

$$
\begin{aligned}
& \mathrm{H}_{(\mathrm{ad})}+\mathrm{H}_{(\mathrm{ad})} \leftrightarrow \mathrm{H}_{2,(g)} ; j_{3}, \\
& \mathrm{H}_{(\mathrm{ad})} \leftrightarrow \mathrm{H}_{(\mathrm{ab})} ; j_{4} .
\end{aligned}
$$

Reaction paths (i)-(iv) state that hydrogen is deposited onto the electrode surface by the first process, the Volmer path, $j_{1}$, and removed by the Heyrovsky-Horiuti, $j_{2}$, the Tafel path, $j_{3}$, and by absorption, $j_{4}$. The absorbed hydrogen is present in the form of protons which at high concentrations may form clusters [4], reactions (v) and (vi), respectively.

$$
\begin{aligned}
& \mathrm{H}_{(\mathrm{ab})} \leftrightarrow \mathrm{H}^{+}{ }_{(r 2)}+\mathrm{e}_{(r 2)}^{-}, \\
& \mathrm{H}_{(\lambda 2)}+\mathrm{H}^{+}{ }_{(\lambda 2)} \leftrightarrow \mathrm{H}^{+} \text {,(ab). }
\end{aligned}
$$

The ionization, (v), and cluster formation, (vi), are considered chemical reactions. However, processes leading to desorption, i.e., the reduction of protons in the reaction layer, $\left(r_{2}\right)$, reactions (vii) and (viii), respectively, are viewed as the solid state analogs of the Volmer and Heyrovsky-Horiuti paths

(vii) $\mathrm{H}_{(r 2)}^{+}+\mathrm{e}_{(r 2)}^{-} \leftrightarrow \mathrm{H}_{(\mathrm{ab})}$,

(viii) $\mathrm{H}_{(r 2)}^{+}+\mathrm{H}_{(\lambda 2)}+\mathrm{e}_{(r 2)}^{-} \leftrightarrow 2 \mathrm{H}_{(\mathrm{ab})}$.

Consequently, while rates of i) and ii) are governed by the Galvani potential, those in the solid-state, (vii) and (viii), depend on the surface potentials [7,8] followed by fast exchange, reaction (iv). We note that in this context an electron is considered either a reactant or product and expressions $\mathrm{e}_{(\lambda 2)}^{-} \rightarrow \mathrm{e}_{(r 1)}^{-} ; \mathrm{e}_{(\lambda 2)}^{-} \rightarrow \mathrm{e}_{(r 2)}^{-}$, represent the same, but energetically different, entities. (Note: Symbols H and D are used interchangeably.)

Transport paths and the resulting structure of the interphase are illustrated in fig. 2upper for absorption, lower for desorption. To reiterate, the cell current, $I$, is split into two streams, $j_{1}$ and $j_{2}(c f$. (i) and (ii)) yielding $\mathrm{H}$ in the adsorbed state that enters the subsurface layer $\left(\lambda_{2}\right)$ at the rate $j_{4}$. The absorbed hydrogen undergoes ionization to $\mathrm{H}^{+}$, (v), followed by diffusion. On desorption, the $\mathrm{H}^{+}$species are transported to the $\left(\lambda_{2}\right)$ layer where they acquire electrons through a chemical recombination reaction, $j_{\text {ch }}$ and an electrochemical step, $j_{\mathrm{el}}$. Subsequently, the neutral species are transferred to the $\left(\lambda_{1}\right)$ layer at the rate $j_{-4}$ followed by oxidation, $j_{-1}$; and reduction and removal, $j_{-2}$, from the electrode surface. This chain of events is not affected by the amount of absorbed hydrogen.

However, kinetic parameters reflecting their mechanistic aspects may substantially vary with the hydrogen content.

3.2. Nature of adsorbed species. - The adsorbed hydrogen, deposited on polarized $\mathrm{Pd}$ surfaces, exists in two forms: the underpotentially adsorbed hydrogen, $\mathrm{H}_{\text {up }}$, with $\Delta \mathrm{G}<0$ and the overpotentially adsorbed, $\mathrm{H}_{\mathrm{op}}$ with $\Delta \mathrm{G}>0$. The $\mathrm{H}_{\text {up }}$ forms a multicoordinated covalent bond with the $\mathrm{Pd}$ and occupies a 3-fold site while the $\mathrm{H}_{\mathrm{op}}$ is singly coordinated with a much weaker bond [11]. Consequently, binding of an atom to a surface involves two subsystems, electronic and ionic, where the contribution of the electronic subsystem to the binding is given by the work function difference. 
One of the, as yet, unresolved problems concerns the species that are transported across the contact surface. Jerkiewicz et al. [12] suggest that $\mathrm{H}_{\mathrm{op}}$ undergoes interfacial transfer. A similar view was expressed by Flitt and Bockris [13], who stated that the low bond energy sites favor absorption. This view is also shared by Chevillot et al. [14]. In their view, the adsorption/absorption can be regarded as, to quote, "the proton passes from one screened system to another, i.e., from the electron screening in the metal to ionic screening of the solvated ion in solution". The strongly adsorbed hydrogen is bound by a quasi-covalence while the weakly adsorbed $\mathrm{H}$ displays a metallic character which favors the equilibrium with the configuration of a proton in solution.

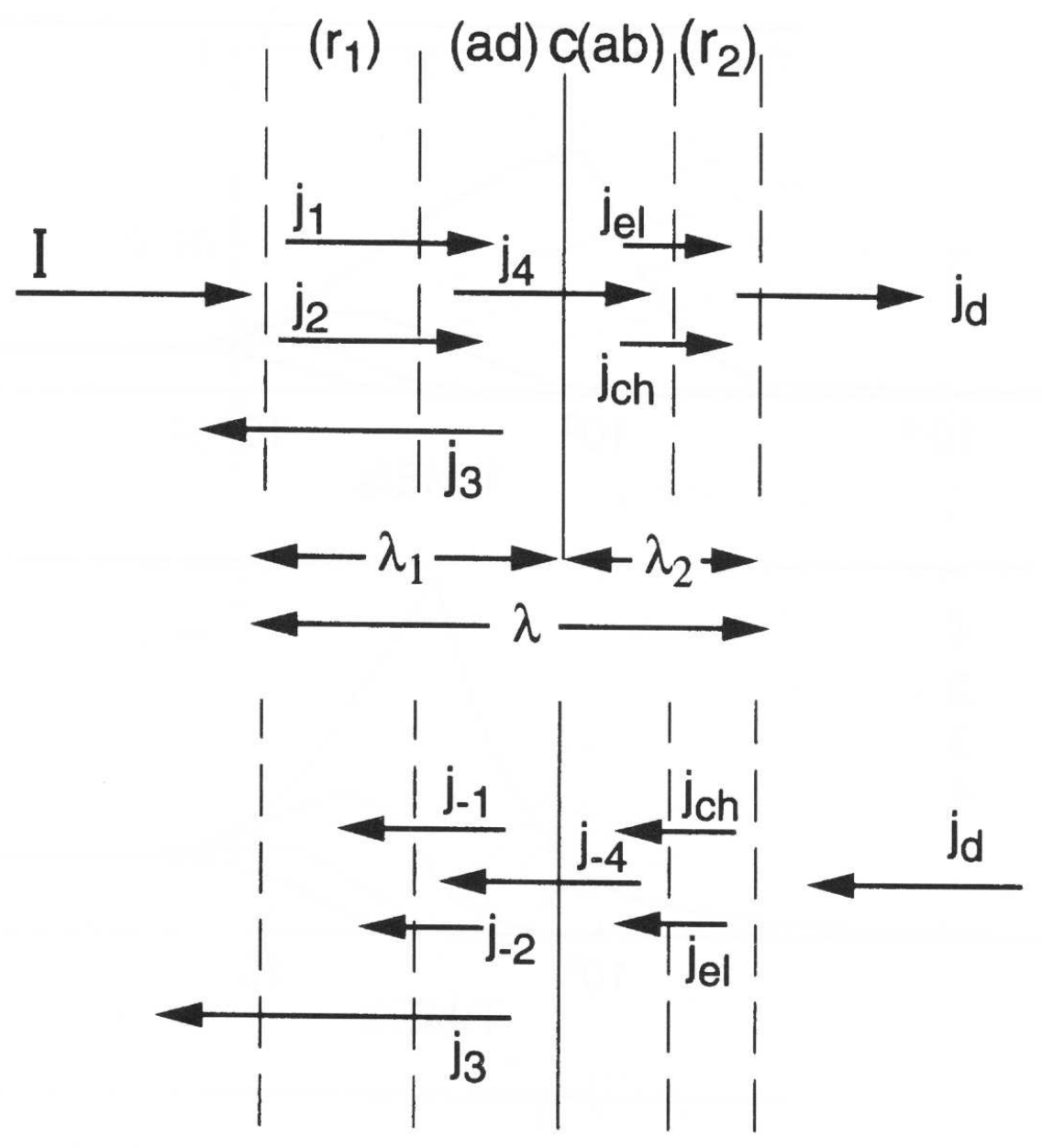

Fig. 2. - Concept of the $\mathrm{Pd} / \mathrm{D}_{2} \mathrm{O}$ interphase: (ad) adsorption layer, (c) contact surface, (ab) absorption layer, $r_{1}$ and $r_{2}$ reaction layers, $b$ and $m$ homogeneous solution and metal phases, respectively. $I$ total current, $j_{1}$ Volmer path, $j_{2}$ Heyrovsky-Horiuti path, $j_{3}$ Tafel path (equivalent), $j_{4}$ and $j_{-4}$ are adsorbedabsorbed exchange equivalent currents. $j_{\mathrm{ch}}$ and $j_{\mathrm{el}}$ are the $\mathrm{H}^{+}$fluxes generated within the subsurface layer, $\left(\lambda_{2}\right)$, by dissociation and oxidation, respectively. $(\lambda)$ is an interphase region consisting of a set of non-autonomous layers $\left(\lambda_{1}\right)=(\mathbf{a d})+\left(r_{1}\right),\left(\lambda_{2}\right)=(\mathbf{a b})+\left(r_{2}\right)$. 
Recent work of Jerkiewicz et al. [12] discussed the structure of the interphase in terms of participating processes. Using our notations, they assumed that the step $\mathrm{H}_{(\mathrm{ad})} \leftrightarrow \mathrm{H}_{(\mathrm{ab})}$ is fast and that the $\mathrm{H}_{(\mathrm{ab})}$ species may be partially ionized. They argued that the surface coverage determines the operating driving force for diffusion and that, because of the fast phase transfer step, the diffusional flux depends on overpotential through $\mathrm{H}_{(\mathrm{ad})} \leftrightarrow \mathrm{H}_{(\mathrm{ab})}$ being in quasi-equilibrium. Jerkiewicz and Zolfaghari [11] added another step in which the $\mathrm{H}^{+} \cdot 4 \mathrm{H}_{2} \mathrm{O}$ species "encounters the region close to the electrode surface where $\mathrm{H}^{+}$ discharge takes place with the formation of absorbed H". In our terminology, this region is defined as the reaction layer, $r_{1}$.

3.3. Development of an active interphase. - The formation, growth and detachment of electrogenerated hydrogen gas bubbles have a profound effect on the overall processes in both the solution and the metal side of the interphase. The evolution of gas bubbles on the electrode surface precludes uniform distribution of the $\mathrm{cd}$ and, therefore, overpotential. This, in turn, results in the removal of an external force acting upon the interphase of the hydrogen-loaded electrode and promotes formation of local gradients, i.e., the formation of conditions believed to initiate the Fleischmann-Pons effect. How deep into the electrode interior these gradients extend depends on the residence time of the gas bubbles and the relaxation time of the processes under consideration.

To illustrate the effect of gas evolution on the redistribution of hydrogen by the exchange between absorbed and adsorbed atoms, one sets the cell current to zero and observes how the system relaxes at, and in the vicinity of, the surface. Using reasonable values for the rate constants of the participating processes [2], the distribution of absorbed deuterium in the first four layers immediately after cell current termination is illustrated in figs. $3 \mathrm{a}, \mathrm{b}$ and c for diffusion coefficients $D=10^{-6}, 10^{-8}$ and $10^{-10} \mathrm{~cm}^{2} \mathrm{~s}^{-1}$ and a charging current of $40 \mathrm{mAcm}^{-2}$ for $\mathrm{t}=1,10$ and 1000 seconds. Inspection of the $\zeta_{i}(t)(i=$ 1,2,3 and 4) curves (fraction of absorbed deuterium) shows that the diffusion coefficient controls the rate and efficiency of absorption, viz., at high diffusion coefficients, e.g., $\mathrm{D}<$ $10^{-8}$, surface processes determine the rate of absorption and the fraction of desorbed hydrogen.

Because of the random distribution of gas bubbles on the electrode surface and the in and out transport of deuterium, situations may arise causing the concentration of absorbed deuterium to be higher in the second layer than in the first layer. The redistribution of deuterium following current termination, fig. 4. indicates the formation of concentration gradients in close proximity to the contact surface. Evidently, the gas evolution contributes to the formation of a zone with time- and space-dependent concentration of deuterium which, in turn, must be considered in the analysis of transport in the bulk material. 

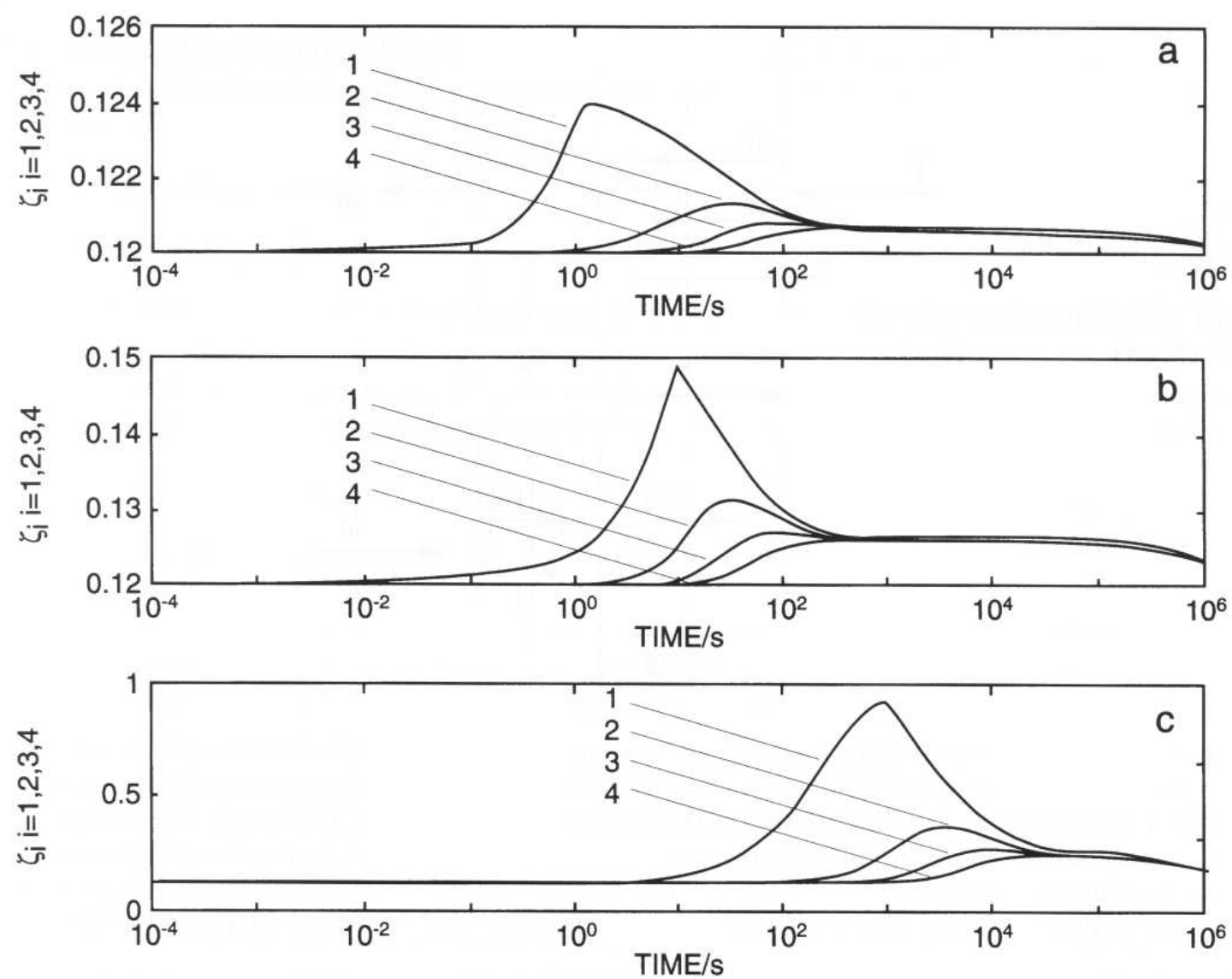

Fig. 3. - Time-dependent distribution of absorbed hydrogen during loading at $I=40 \mathrm{mAcm}^{-2}$ and unloading for: a) $D=10^{-6} \mathrm{~cm}^{2} \mathrm{~s}^{-1}$; b) $10^{-8} \mathrm{~cm}^{2} \mathrm{~s}^{-1}$ and $D=10^{-10} \mathrm{~cm}^{2} \mathrm{~s}^{-1}$. Cell current terminated at 1,10 and 1000 seconds, respectively. Rate constants: $k_{1}=10^{3} \mathrm{~cm}^{3} \mathrm{~mol}^{-1}, k_{2}=10^{2} \mathrm{~cm}^{3} \mathrm{~mol}^{-1}, k_{3}=10^{3} \mathrm{~cm}^{2} \mathrm{~mol}^{-1}$, $k_{4}=2 \times 10^{5} \mathrm{~cm}^{3} \mathrm{~mol}^{-1} \mathrm{~s}^{-1}$ and $k_{d}=2 \times 10^{-2} \mathrm{~cm}^{4} \mathrm{~mol}^{-1} \mathrm{~s}^{-1}$. 


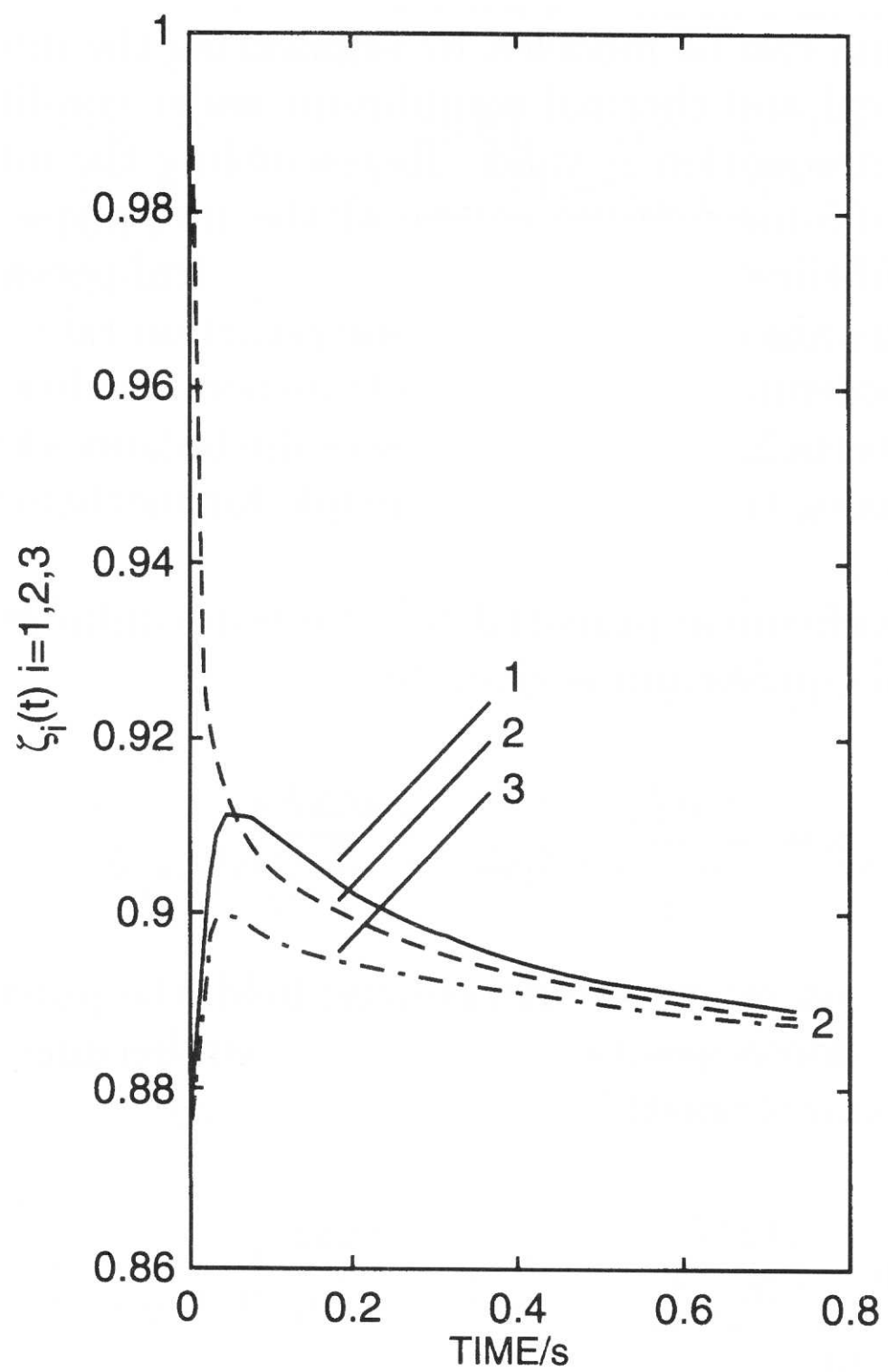

Fig. 4. - Time-dependent distribution of absorbed hydrogen following cell current termination. Rate constants as in fig. 3. Assumed highest concentration in the second layer.

3.4. Interphase relaxation. - The electrode/electrolyte interphase is a non-electroneutral entity. The application of an external field further magnifies the non-electroneutral character. In particular, any change in the applied external field will greatly affect events occurring in the interphase region. It is within this environment that the effect of gas evolution on the interphase events should be discussed. The consequence of gas evolution is a localized change in the electrode potential which, in turn, changes the magnitude of the operating driving forces, i.e., chemical/electrochemical potentials.

The use of the chemical potential as the driving force for the transport of species between two phases is subject to the application of the Gibbs-Duhem equation, i.e., the requirenment that the temperature and concentration can be defined, or that local equilibrium can be assumed, which means that "local extensive property is the same 
function of the local macroscopic variables as at equilibrium" [15]. But the use of the electrochemical potential as the driving force for, e.g., transport across the interphase or other processes, puts the system in a non-equilibrium state. A non-equilibrium interphase in local equilibrium can be modeled by segmenting the interphase into individual layers, each in mechanical and thermal equilibrium under conditions of transport, i.e., where the Gibbs-Duhem equation is valid. Reassembling the interphase and assuming local equilibria, the non-homogeneous nature of the interphase is restored. While in each layer the Gibbs-Duhem equation is valid, the chemical potentials of the $i$-th species in the neighboring layers are different. Transport/reaction rates are determined by the difference in chemical potentials or, in the "no transport condition", by the acting fictitious forces. Thus, the term $\Sigma n_{i}, \mathrm{~d} \mu_{i}=0$ expresses the balance of thermodynamic forces, i.e., it represents an analog to d'Alembert principle for mechanical system and not the GibbsDuhem equation.

The chemical/electrochemical potential in a system containing charged particles in mechanical and thermal equilibrium is given by

$$
\mu=\left.\frac{\partial \Delta G}{\partial n_{i}}\right|_{p, T, n_{i} \neq n_{j}}=\left.\frac{\partial \Delta F}{\partial n_{i}}\right|_{V, T, n_{j} \neq n_{i}} .
$$

When this system is placed in an external electric field, the potential energy of charged particles becomes a function of position, i.e., the system becomes non-electroneutral. In this case, the electrochemical potential is given by

$$
\mu=\left.\frac{\partial \Delta G}{\partial n_{i}}\right|_{p, T, n_{i} \neq n_{j}, \psi}=\left.\frac{\partial \Delta F}{\partial n_{i}}\right|_{V, T, n_{j} \psi \neq n_{i}},
$$

where $\psi$ is the external potential arising from uniform distribution of charges on the contact surface. The electrochemical potential of any charged species present in a thin layer can be evaluated by taking the derivative of the Gibbs (Helmholtz) free energy while keeping the temperature, pressure (volume), mole number and the externally applied potential constant [16].

A change in the electrochemical potential affects the species present at the contact surface as well as charged species in the absorbed state in close proximity to the contact surface. To assess qualitatively the ensuing changes, it is convenient to consider the structure of the interphase and the processes that occur as it transits from one "stopped condition" to another, the relaxation of the interphase. The complex nature of the interphase arises not only from the processes (i)-(viii) but also from changing volume and/or pressure. Thus, we can visualize a relaxation spectrum which leads to the development of gradients. Furthermore, a rapid change in the chemical potential may affect the kinetics of transformation, e.g., diffusional transport from that described by the usual parabolic equation to hyperbolic one [17].

\section{4. - Concluding remarks}

1) Unlike joule heating, excess enthalpy generation occurs in the form of localized events in close proximity to the contact surface. 
2) The higher the electrolyte temperature, the more frequent the events. In the limit, these events overlap to produce oscillating islands.

3) Although gas evolution is not the primary reason for the initiation of the thermal events, it substantially modifies the structure of the interphase.

$$
* * *
$$

The authors wish to express their gratitude to Dr. F. Gordon, Spawar Systems Center San Diego, Code D30 for his interest, encouragement and support.

\section{REFERENCES}

[1] Fleischmann M., Pons S. and Hawkins M., J. Electroanal. Chem., 261 (1989) 301.

[2] Szpak S., Gabriel C. J., Smith J. J. and Nowak R. J., J. Electroanal. Chem., 309 (1991) 273.

[3] Szpak S., Mosier-Boss P. A., Gabriel C. J. and Smith J. J., J. Electroanal. Chem., 365 (1994) 275.

[4] Szpak S., Mosier-Boss P. A., Scharber S. R. and Smith J. J., J. Electroanal. Chem., 337 (1992) 147.

[5] Kopper M. T. M., Electrochim. Acta, 37 (1992) 1771.

[6] Kopper M. T. M. and Sluyters J. H., Electrochim. Acta, 38 (1993) 1535.

[7] Fleischmann M., Pons S., LeRous M. and Roulette J., Trans. Fusion Technol., 26 (1994) 323.

[8] Pons S. and Fleischmann M., J. Chim. Phys., 93 (1996) 711.

[9] Szpak S. and Mosier-Boss P. A., Phys. Lett. A, 221 (1996) 141.

[10] Van Rysselberghe P., Some Aspects Of The Thermodynamic Structure Of

Electrochemistry, in Modern Aspects Of Electrochemistry, Vol. 4 (Plenum Press, New York) 1966.

[11] Jerkiewicz G. and Zolfaghari A., J. Electrochem. Soc., 143 (1996) 1240.

[12] Jerkiewicz G., Borodzinski J. J., Chrzanowski W. and Conway B. E., J.

Electrochem. Soc., 142 (1995) 3755.

[13] Flitt H. J. and Bockris J. O’M., J. Hydrogen Energy, 7 (1982) 411.

[14] Chevillot J.-P., Farcy J., Hinnen C. and Roussau A., J. Electroanal. Chem., 64 (1975) 39.

[15] Defay R., Prigogine I. and Bellemans A., Surface Tension and Adsorption

(Longmans, Green and Co, Ltd, London W1) 1951.

[16] Sorensen T. B. and Compan V., Electrochim. Acta, 42 (1997) 639.

[17] Sobolev S. L., Technical Physics, 43 (1998) 307. 